\title{
Ion extraction from carbon shunting arc plasma
}

\author{
K. Takaki, ${ }^{\text {a) }}$ S. Mukaigawa, and T. Fujiwara \\ Department of Electrical and Electronic Engineering, Iwate University, 4-3-5 Ueda, Morioka, \\ Iwate 020-8551, Japan \\ M. Kumagai and K. Yukimura \\ Department of Electrical Engineering, Doshisha University, 1-3 Tatara-Miyakodani, Kyotanabe, \\ Kyoto 610-0321, Japan
}

(Received 27 December 2002; accepted 28 February 2003)

\begin{abstract}
The ion current characteristics of the shunting arc discharge are described in this letter. A carbon rod with $2 \mathrm{~mm}$ diameter and $40 \mathrm{~mm}$ length was employed for arc generation. The combination of the shunting arc and the negative pulse voltage applied to target is promising for plasma-based ion implantation and deposition for metallic or semimetallic three-dimensional materials. The delay time, which is defined as the time between the start of the arc current and applying the pulse voltage, was varied. The ions are extracted from the shunting arc plasma by applying a pulsed bias voltage to a target set nearby the arc source. The arc current lasts $40 \mu \mathrm{s}$, of which peak is $1.7 \mathrm{kA}$. The extracted target current has a sharp peak at the initial stage, followed by a stationary state. The stationary current decreases with increasing the delay time and increases with increasing the bias voltage. Under the assumption of a collisionless ion sheath, the plasma density was estimated. At the boundary between the ion sheath and the shunting arc plasma, the density was approximately 5 $\times 10^{8} \mathrm{~cm}^{-3}$ and $3 \times 10^{7} \mathrm{~cm}^{-3}$ for the delay times of 100 and $1000 \mu$ s, respectively. The stationary ion current linearly increases with increasing the bias voltage and can be expressed in a simple equation. (C) 2003 American Institute of Physics. [DOI: 10.1063/1.1568822]
\end{abstract}

Plasma-based ion implantation and deposition (PBI\&D) is promising for improving surface properties of threedimensional substrates as a workpiece. ${ }^{-1,2}$ A negative pulse voltage is applied to the workpiece immersed in a plasma, around which a transient ion sheath is made to extract ions. The ions are implanted into and/or deposited on the workpiece. Generally, various kinds of workpieces are used for modification using the PBII\&D technique. ${ }^{3,4}$. As an example of the nonmetallic substrates is a polyethylene terepthalate film, which is modified to suppress the oxygen transmission. ${ }^{4}$

The shunting arc, which is a pulsed plasma source for metallic and nonmetallic materials such as carbon and tungsten, is characterized by self-ignition with heating a rod. The principle of the shunting arc generation was described elsewhere. ${ }^{5}$ The shunting arc system consists of a simple construction and therefore, easy handling to be cost-effective instrumentation is possible. ${ }^{6-8}$ The arc plasma is generated using a gentle evaporation from the rod heated by a pulsed current. Accordingly, the shunting arc is promising to prepared droplet-free films.

It has been known that the arc expands toward the radial direction with a speed over $20 \mathrm{~km} / \mathrm{s}$. Due to a fast-expanding and pulsed plasma, it was difficult to measure the plasma density, experimentally. In this letter, the shunting arc plasma density near the sheath around the negatively biased target was calculated using voltage-current characteristics under the simple assumption.

A schematic diagram of the experimental facilities is shown in Fig. 1. A cylindrical vacuum chamber with $600 \mathrm{~mm}$ long and $500 \mathrm{~mm}$ inner diameter was used with a turbomo-

a)Electronic mail:.takaki@iwate-u.ac.jp lecular pump and two rotary pumps for evacuation to 6.7 $\mathrm{mPa}$ as a base pressure. $\mathrm{A} 40 \mathrm{~mm}$ long carbon rod (C: $97.3 \%$, O: $2.5 \%$, Al: $0.1 \%$, Si: $0.12 \%$ ) with $2 \mathrm{~mm}$ diameter was held horizontally by a pair of carbon electrodes. The resistances of the rod are approximately 1.0 and $15.8 \Omega$ per $40 \mathrm{~mm}$. A 20

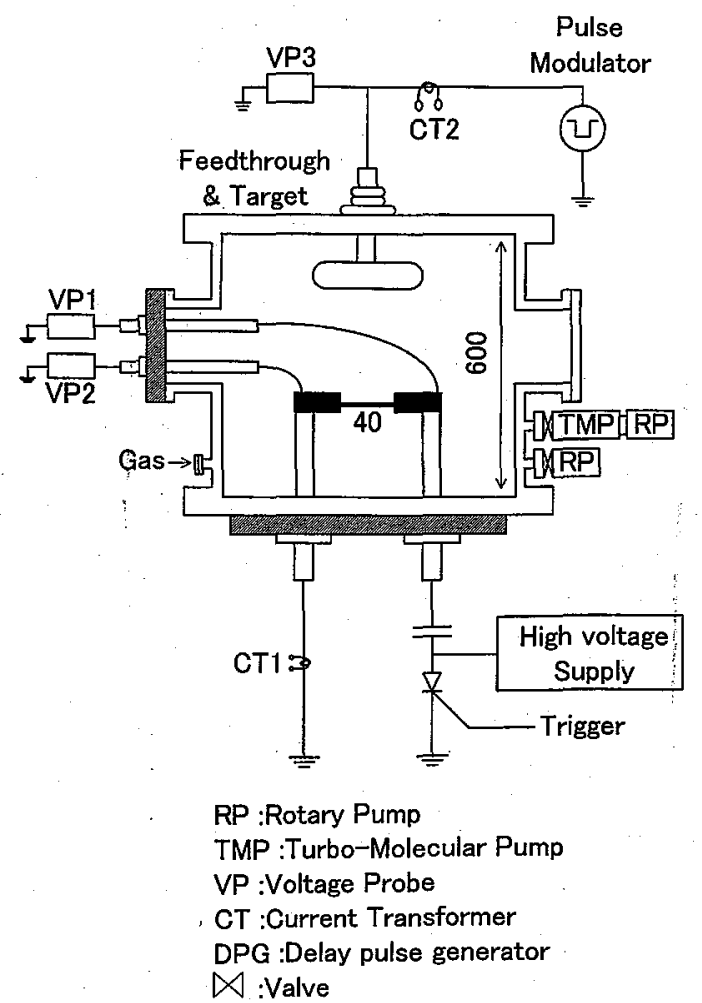

FIG. 1. A schematic diagram of the experimental facilities used for shunting arc generation and the ion extraction. 


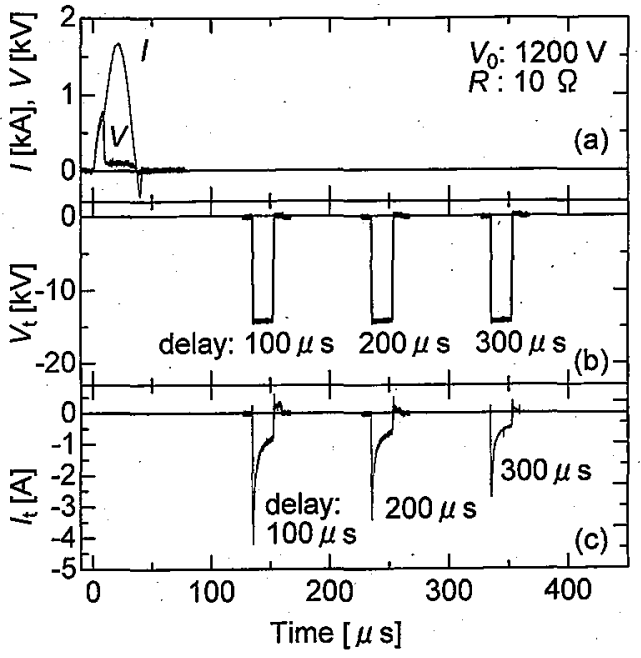

FIG. 2. Wave forms of: (a) a current through the electrodes and a voltage between the electrodes, (b) applied voltages to the target, and (c) currents of the target for various delay times from arc initiation (rod resistance: $1.0 \Omega$, bias voltage: $-15 \mathrm{kV}$ ).

$\mu \mathrm{F}$ capacitor bank was used to initiate a shunting arc using a thyristor as a closing switch and a charging voltage of the capacitor was $1200 \mathrm{~V}$.

A $60 \mathrm{~mm}$ diameter disk target with $11 \mathrm{~mm}$ thickness was positioned at about $75 \mathrm{~mm}$ away from the carbon rod. A series of negative voltage of an amplitude up to $-40 \mathrm{kV}$ with a pulse width of $20 \mu \mathrm{s}$ is applied to the target with a repetition rate of $5 \mathrm{kHz}$.

Wave forms of the bias voltage and currents through the modulator and arc generation circuit were simultaneously monitored by an oscilloscope. The wave form of the voltage across the rod was observed in a differential mode using two voltage probes, VP1 and VP2 shown in Fig. 1.

Figure 2(a) shows wave forms of the rod current $I$ and rod voltage $V$ at an ambient pressure of $6.7 \mathrm{mPa}$. The rod voltage is divided into two regions, i.e., the initial stage in a rising portion of the voltage and the other appears following the initial stage and has a constant voltage of about $100 \mathrm{~V}$. These correspond to the rod heating and a shunting arc discharge, respectively. ${ }^{6}$ The arc current shows a sinusoidal wave form due to a resonance between the capacitance and the circuit inductance. It is seen that the peak current of the arc discharge is about $1.7 \mathrm{kA}$.

The target current by applying pulse bias voltage of $-15 \mathrm{kV}$ has a sharp peak, which appears almost simultaneously at the voltage application, followed by a stationary state. As seen from Figs. 2(b) and 2(c), the stationary current decreases with increasing the delay time. Both values of the peak and subsequent stationary currents decrease with the delay time, which implies the decrease of the plasma density. However, the target current can be observed even at a delay time of $1000 \mu \mathrm{s}$.

The target current $I_{t}$ consists of three components as shown in Eq. (1):

$$
I_{t}=\frac{d}{d t}[c(t) \cdot v(t)]+\left(1+\gamma_{i}\right) I_{i}
$$

where $c$ is the capacitance of the sheath around the target, $v$ is the applied bias voltage, and $\gamma_{i}$ is the secondary electron

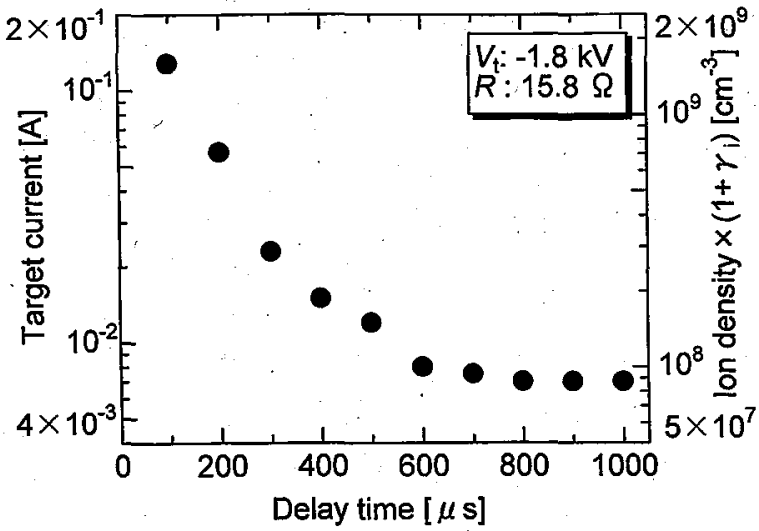

FIG. 3. Ion density multiplied by $\left(1+\gamma_{i}\right)$ as function of the delay time (rod resistance: $15.8 \Omega$, bias voltage: $-1.8 \mathrm{kV}$ ).

emission coefficient by an ion impact. An initial peak in the target current is based on the capacitive component.

Figure 3 shows the stationary current as a function of delay time at bias voltage of $-1.8 \mathrm{kV}$. The rod resistance is $15.8 \Omega$, respectively. It is seen that the stationary current decreases with the delay time. The extracted ion current $I_{i}$ can be expressed as Eq. (2):

$$
I_{i}=n_{i} \cdot e \cdot v_{i} \cdot S \text {, }
$$

where $n_{i}$ is the ion density, $e$ is the elementary charge, $v_{i}$ is the ion velocity, and $S$ is the surface area of the target. Assuming that the ion flow is collisionless, $v_{i}$ at the target can be expressed as Eq. (3):

$$
v_{i}=\sqrt{\frac{2 e V}{m_{i}}}
$$

where $m_{i}$ is the ion mass and $V$ is the applied target voltage. The ion density $n_{i}$ multiplied by $\left(1+\gamma_{i}\right)$ is also shown as a vertical axis. $\gamma_{i}$ can be roughly estimated for ion energy $E$ by Eq. (4):

$$
\gamma_{i}=0.052 E^{1 / 2}
$$

which was obtained for $\mathrm{N}_{2}^{+}$ion beam experiment for a stainless-steel target in an independent high vacuum $(<0.001 \mathrm{~Pa}) .{ }^{9}$ When the ion energy is assumed to be equal to the target bias, $\gamma_{i}$ is estimated as 2.2 at a target bias of $-1.8 \mathrm{kV}$. Using Eqs. (2)-(4), the ion density around the target can be calculated to $5 \times 10^{8} \mathrm{~cm}^{-3}$ for the delay time of $100 \mu \mathrm{s}$. The ion density decreases to $3 \times 10^{7} \mathrm{~cm}^{-3}$ with increasing the delay time from 100 to $1000 \mu \mathrm{s}$.

When the bias voltage is varied up to $-40 \mathrm{kV}$, the stationary current $I_{s}$ increases with increasing the voltage. Figure 4 shows $I_{s}$ as a function of bias voltage at a delay time of $250 \mu \mathrm{s}$. The charging voltage of the capacitor and the initial resistance of the rod are $1200 \mathrm{~V}$ and $1.0 \Omega$, respectively. Because the displacement current shown in the first term of the right-hand side of Eq. (1) is negligible in the stationary state, $I_{s}$ can be expressed as follows:

$$
I_{s}=\left(1+\gamma_{i}\right) \cdot n_{i} \cdot e \cdot S \cdot \sqrt{\frac{2 e V}{m_{i}}}
$$

Under the assumption of the collisionless ion flow, Eq. (5) can be rewritten as Eq. (6): 


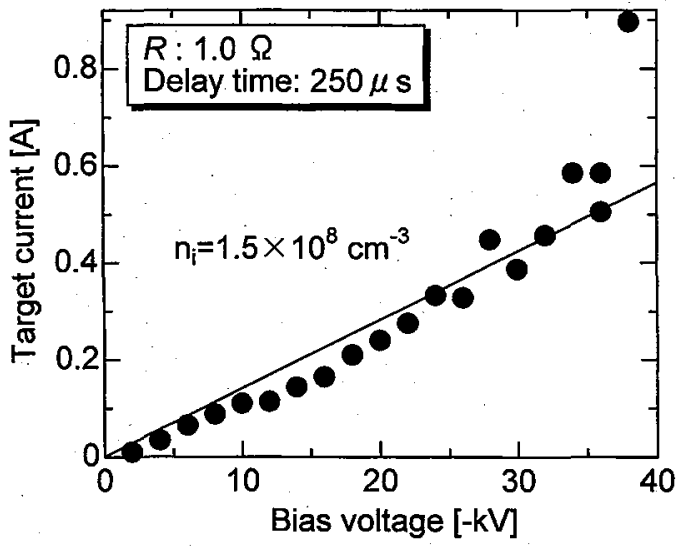

FIG. 4. Stationary current at end of the pulse as function of amplitude of the bias voltage (rod resistance: $1.0 \Omega$, delay time: $250 \mu \mathrm{s}$ ).

$$
I_{s}=n_{i} \cdot \sqrt{\frac{2}{m_{i}}} \cdot e^{1.5} \cdot S \cdot(1+0.052 \sqrt{V}) \cdot \sqrt{V}
$$

If $1 \ll 0.052 \sqrt{\nabla}$, Eq. (6) can be rewritten to a simple form of the equation as shown in Eq. (7):

$$
I_{s}=0.052 \sqrt{2} \cdot n_{i} \cdot m_{i}^{-0.5} \cdot e^{1.5} \cdot S \cdot V .
$$

A solid straight line in Fig. 4 shows a fitting result of the target current using Eq. (7), from which $n_{i}$ near the target is estimated as $1.5 \times 10^{8} \mathrm{~cm}^{-3}$. The values of $S=28.3 \mathrm{~cm}^{2}$, $m_{i}=2 \times 10^{-26} \mathrm{~kg}, e=1.6 \times 10^{-19} \mathrm{C}$ are used in this calculation. The ion density of $1.5 \times 10^{8} \mathrm{~cm}^{-3}$ for a $1.0 \Omega$ carbon rod is lower than that of $2.8 \times 10^{8} \mathrm{~cm}^{-3}$ for a $15.8 \Omega$ carbon rod at a delay time of $250 \mu \mathrm{s}$. The heating energy consumed by a $1.0 \Omega$ carbon rod is larger than that of a $15.8 \Omega$ carbon rod under the present experimental condition. As the results, the arc current of a $1.0 \Omega$ carbon rod is lower than that of a 15.8 $\Omega$ carbon rod at same charging voltage of the capacitor, ${ }^{10}$ which is probably due to ion density of a $1.0 \Omega$ carbon rod lower than that of a $15.8 \Omega$ carbon rod.

${ }^{1}$ R. J.Adler and S. T. Picraux, Nucl. Instrum. Methods Phys. Res. B 6, 123 (1985).

${ }^{2}$ J. R. Conrad, J. L. Radtke, R. A. Dodd, F. J. Worzala, and N. C. Tran, J. Appl. Phys. 62, 4591 (1987).

${ }^{3}$ W. Ensinger, K. Volz, and B. Enders, Surf. Coat. Technol. 136, 202 (2001).

${ }^{4}$ M. Yoshida, T. Tanaka, M. Shinihara, S. Watanabe, J. W. Lee, and T. Takagi, J. Vac. Sci. Technol. A 20, 1 (2002).

${ }^{5} \mathrm{~K}$. Yukimura and S. Masamune, Surf. Coat. Technol. 156, 31 (2002).

${ }^{6} \mathrm{~K}$. Yukimura and S. Masamune, Rev. Sci. Instrum. 73, 860 (2002).

${ }^{7}$ K. Yukimura, R. Isano, T. Monguchi, and K. Yoshioka, J. Vac. Sci. Technol. B 17, 871 (1999).

${ }^{8} \mathrm{~K}$. Yukimura and Y. Tani, Surf. Coat. Technol. 142-144, 388 (2001).

${ }^{9}$ J. Brutscher, R. Günzel, and W. Möller, Plasma Sources Sci. Technol. 5, 54 (1995).

${ }^{10}$ K. Takaki, S. Mukaigawa, T. Fujiwara, M. Kumagai, and K. Yukimura, Nucl. Instrum. Methods Phys. Res. B (to be published). 